\title{
Superparamagnetism-induced mesoscopic electron focusing in topological insulators
}

\author{
P. Sessi, ${ }^{1,}{ }^{*}$ P. Rüßmann, ${ }^{2}$ T. Bathon, ${ }^{1}$ A. Barla,${ }^{3}$ K. A. Kokh, ${ }^{4,5}$ O. E. Tereshchenko, ${ }^{5,6}$ K. Fauth, ${ }^{1,7}$ S. K. Mahatha, ${ }^{3}$ \\ M. A. Valbuena, ${ }^{8}$ S. Godey, ${ }^{8}$ F. Glott,${ }^{1}$ A. Mugarza,${ }^{8,9}$ P. Gargiani,${ }^{10}$ M. Valvidares,${ }^{10}$ N. H. Long, ${ }^{2}$ \\ C. Carbone ${ }^{3}$ P. Mavropoulos, ${ }^{2}$ S. Blügel,${ }^{2}$ and M. Bode ${ }^{1,7}$ \\ ${ }^{1}$ Physikalisches Institut, Experimentelle Physik II, Universität Würzburg, Am Hubland, 97074 Würzburg, Germany \\ ${ }^{2}$ Peter Grünberg Institut and Institute for Advanced Simulation, Forschungszentrum Jülich and JARA, 52425 Jülich, Germany \\ ${ }^{3}$ Istituto di Struttura della Materia, Consiglio Nazionale delle Ricerche, 34149 Trieste, Italy \\ ${ }^{4}$ V.S. Sobolev Institute of Geology and Mineralogy, Siberian Branch, Russian Academy of Sciences, 630090 Novosibirsk, Russia \\ ${ }^{5}$ Novosibirsk State University, 630090 Novosibirsk, Russia \\ ${ }^{6}$ A.V. Rzanov Institute of Semiconductor Physics, Siberian Branch, Russian Academy of Sciences, 630090 Novosibirsk, Russia \\ ${ }^{7}$ Wilhelm Conrad Röntgen-Center for Complex Material Systems (RCCM), Universität Würzburg, Am Hubland, 97074 Würzburg, Germany \\ ${ }^{8}$ Catalan Institute of Nanoscience and Nanotechnology (ICN2), CSIC, \\ and The Barcelona Institute of Science and Technology, Bellaterra, 08193 Barcelona, Spain \\ ${ }^{9}$ ICREA-Institucio Catalana de Recerca i Estudis Avancats, Lluis Companys 23, 08010 Barcelona, Spain \\ ${ }^{10}$ ALBA Synchrotron Light Source, 08290 Cerdanyola del Vallès, Barcelona, Spain \\ (Received 1 March 2016; revised manuscript received 27 July 2016; published 17 August 2016)
}

\begin{abstract}
Recently it has been shown that surface magnetic doping of topological insulators induces backscattering of Dirac states which are usually protected by time-reversal symmetry [Sessi et al., Nat. Commun. 5, 5349 (2014)]. Here we report on quasiparticle interference measurements where, by improved Fermi level tuning, strongly focused interference patterns on surface $\mathrm{Mn}$-doped $\mathrm{Bi}_{2} \mathrm{Te}_{3}$ could be directly observed by means of scanning tunneling microscopy at $4 \mathrm{~K}$. Ab initio and model calculations reveal that their mesoscopic coherence relies on two prerequisites: (i) a hexagonal Fermi surface with large parallel segments (nesting) and (ii) magnetic dopants which couple to a high-spin state. Indeed, x-ray magnetic circular dichroism shows superparamagnetism even at very dilute Mn concentrations. Our findings provide evidence of strongly anisotropic Dirac-fermion-mediated interactions and demonstrate how spin information can be transmitted over long distances, allowing the design of experiments and devices based on coherent quantum effects in topological insulators.
\end{abstract}

DOI: 10.1103/PhysRevB.94.075137

Coherence is a general property of waves which describes the capability of keeping a well-defined phase relation while propagating in space and time. Because of the particle-wave duality, which lies at the very foundation of quantum mechanics, the same concept can also be applied to quasiparticles in solids. Quantum coherence is of fundamental importance since it sets the limits up to which information can be transmitted and processed with high fidelity. With the invention of scanning tunneling microscopy (STM), it became possible to visualize coherent phenomena in real space by imaging the standing wave pattern produced by scattering events around individual atomic-scale defects [1]. The further development of STM made it possible to engineer properties at the atomic scale. This capability was used for the creation of quantum mirages [2], for extracting the phase of electron wave functions [3], and for visualizing the indirect coupling mechanisms, such as the Ruderman-Kittel-Kasuya-Yosida (RKKY) interaction [4]. More recently, quasiparticle interference (QPI) imaging has made it possible to analyze how propagating waves in solids are influenced by the periodic potential of the crystal lattice. In particular, it has been shown that quasiparticle waves propagate anisotropically when the shape of a constant-energy cut (CEC) deviates from an isotropic contour. In analogy to optics, focusing and defocusing lead to an enhanced intensity along certain crystallographic directions and to partial or even complete suppression along others, respectively [5]. However,

*Corresponding author: sessi@physik.uni-wuerzburg.de despite its relevance in several areas of modern condensed matter, such as spintronics and quantum computation, the role played by the spin degree of freedom has not yet been explored.

Within this framework, topological insulators (TIs) represent a promising class of materials. TIs are insulating in the bulk but conducting on the surface, where they host linearly dispersing massless fermions [6]. The strong spinorbit coupling locks spin and momentum, thereby suppressing backscattering $[7,8]$, and results in spin currents which are intrinsically tied to charge currents [9]. The overwhelming majority of studies have discussed electronic states in the vicinity of the Dirac point, where the isotropic linear band dispersion relation results in a circular CEC. It is well known, however, that CECs progressively evolve from an almost circular (convex) shape close to the Dirac energy $E_{\mathrm{D}}$ to a concave, snowflakelike shape at higher energies $[10,11]$. Once this transition takes place, large parallel sections face each other, a scenario supporting good nesting vectors which can strongly enhance the susceptibility to external perturbations.

In pristine TIs, these scattering vectors are suppressed by time-reversal symmetry [7,8]. Recently, it has been shown by QPI experiments, for both bulk-doped [12] as well as for surface-doped TIs [13], that the introduction of magnetic species breaks time-reversal symmetry, lifts this protection, and opens the backscattering channel. In any case, however, the signal along the $\overline{\Gamma K}$ direction characteristic for backscattering was either very weak [12] or comparable to other scattering channels [13]. Here, by carefully tuning the Fermi level of a magnetically doped TI surface, we maximize backscattering 
such that it leads to highly focused interference patterns supported by good nesting conditions. The resulting coherent quantum oscillations can be observed by QPI over distances of tens of nanometers without any significant intensity loss. Theoretical calculations show that long coherence lengths require two conditions to be fulfilled: (i) the Fermi surface (FS) must exhibit large parallel segments (nesting) and (ii) the magnetic dopants must couple ferromagnetically (FM) to create a highspin state. As revealed by $\mathrm{x}$-ray magnetic circular dichroism (XMCD) measurements, superparamagnetic coupling already emerges at very dilute $\mathrm{Mn}$ concentrations on $\mathrm{Bi}_{2} \mathrm{Te}_{3}$. Our results suggest that-by appropriate band engineeringspin-dependent quantum coherent transport can be achieved over mesoscopic distances with potential applications in novel device concepts that rely on quantum coherent effects in TIs.

STM and XMCD experiments were performed at $T<5 \mathrm{~K}$. In either case, $\mathrm{Mn}$ was deposited by electron-beam evaporation onto the cold sample surface $(T \leqslant 7 \mathrm{~K})$. The deposition rate was calibrated by topographic STM images or based on X-ray absorption spectra (XAS) at the $\mathrm{Mn} L_{2,3}$ edges. Coverage uncertainty is about $15 \%$. In order to determine the Mn distribution on the surface, we have performed an analysis of STM images regarding nearest-neighbor adatom distances (see Supplemental Material [14]). The distribution peaks at an interatomic distance between 1 and $3 \mathrm{~nm}$, but occasionally Mn atoms in very close proximity below $5 \AA$ can be found.

Figure 1(a) shows the constant-current STM image of a $\mathrm{Bi}_{2} \mathrm{Te}_{3}$ crystal which was surface doped with $\mathrm{Mn}$. The nominal Mn coverage amounts to 0.01 monolayer (ML) and gives rise to a rigid negative-energy shift of $\approx 120 \mathrm{meV}$, resulting in an $n$-doped surface with $E_{\mathrm{D}} \approx-250 \mathrm{meV}$ below the Fermi level $E_{\mathrm{F}}$ [13]. Note that this leads to $E_{\mathrm{F}}$ positioned inside the bulk band gap, making the Fermi sea a truly twodimensional system (see Supplemental Material [14]). The dispersion above the Dirac point is schematically represented in Fig. 1(b). Selected CECs relevant to the following discussion are sketched in Figs. 1(c)-1(e). Just above $E_{\mathrm{D}}$ [Fig. 1(e)], the $\mathrm{CEC}$ is circular (black line) and the spin is perpendicularly locked to the momentum, thereby leading to a helical spin structure indicated by colorized arrows. As we move further away from the Dirac point, warping increases. This first leads to a hexagonal [Fig. 1(d)] and eventually to a snowflakelike shape [Fig. 1(c)] of the CEC. This deformation goes along with the development of an alternating out-of-plane component of the spin polarization along the $\overline{\Gamma K}$ directions of the surface Brillouin zone [11], indicated by symbols $(\otimes, \odot)$ in Figs. 1(c) and $1(\mathrm{~d})$.
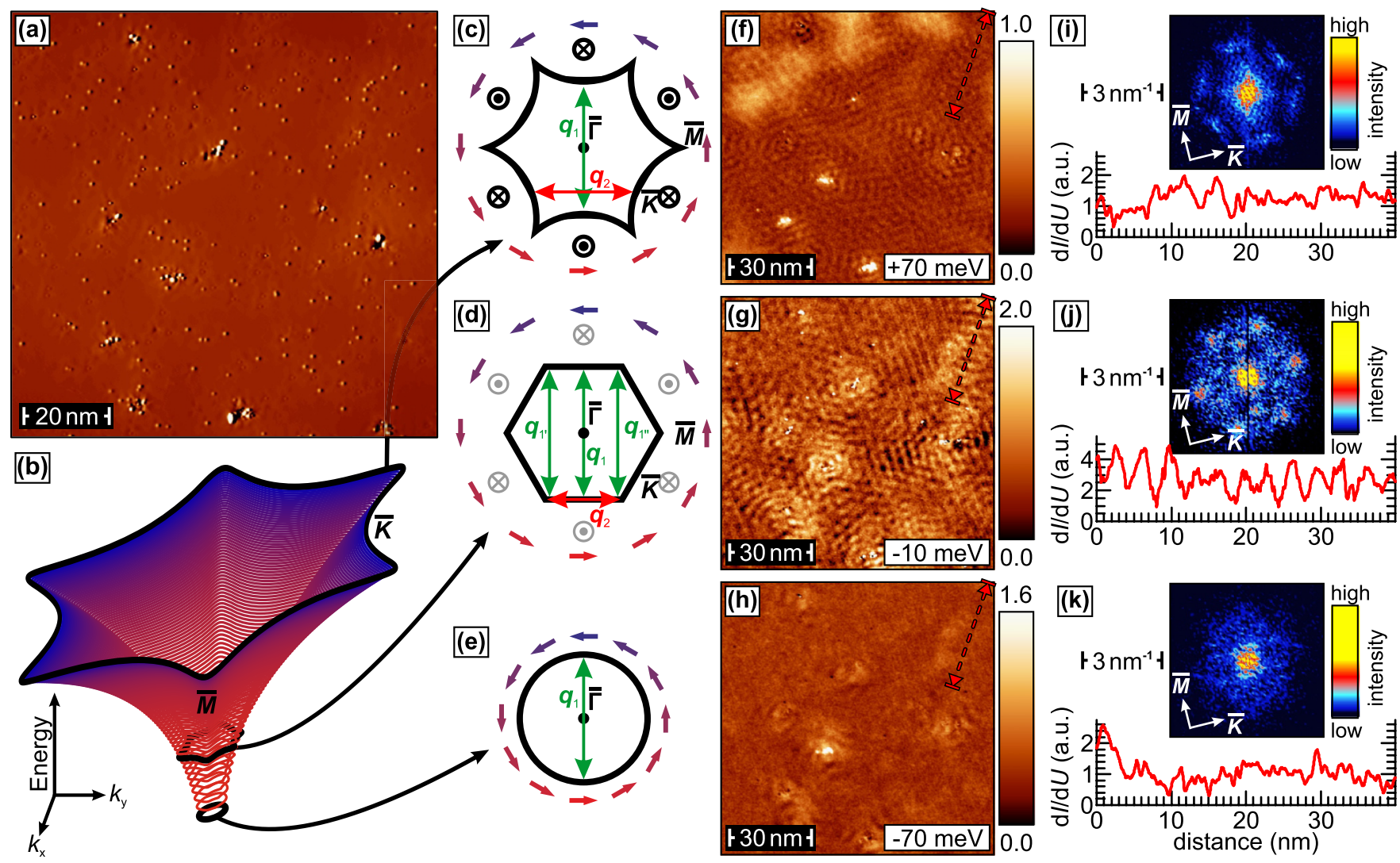

FIG. 1. (a) Topographic STM image of $0.01 \mathrm{ML} \mathrm{Mn} \mathrm{on} \mathrm{Bi}_{2} \mathrm{Te}_{3}(I=35 \mathrm{pA} ; U=150 \mathrm{mV})$. (b) Schematic dispersion above the Dirac point and (c)-(e) constant-energy contours with in-plane/out-of-plane spin polarization at selected energies. Green and red arrows indicate preferred scattering vectors $\mathbf{q}_{1}$ and $\mathbf{q}_{2}$ along the $\overline{\Gamma K}$ and $\overline{\Gamma M}$ directions, respectively. Corresponding quasiparticle interference maps measured at energies that mark the transition from convex to concave constant-energy cuts are shown in (f) $E-E_{\mathrm{F}}=+70 \mathrm{meV}$, (g) $-10 \mathrm{meV}$, and (h) $-70 \mathrm{meV}$. Line sections measured between the arrows are plotted in panels (i)-(k). Insets show the corresponding Fourier-transformed $d I / d U$ maps (top, raw data). 
Figures 1(f)-1(h) show QPI maps measured at $E-E_{\mathrm{F}}=$ $+70 \mathrm{meV},-10 \mathrm{meV}$, and $-70 \mathrm{meV}$, respectively, marking the transition from convex to concave CEC, as shown theoretically [11] and experimentally [10]. As indicated by green and red arrows in Figs. 1(c)-1(e), the scattering vectors $\mathbf{q}_{1}$ and $\mathbf{q}_{2}$ specific for each energy can be obtained by using the stationary phase approximation [15]. At $E-E_{\mathrm{F}}=-70 \mathrm{meV}$ [Figs. 1(e) and 1(h)], the CEC is almost circular. Since reasonably nested parts of the CEC essentially contain only one scattering vector $\mathbf{q}_{1}$, only very weak QPI modulations are observed in Fig. 1(h). Indeed, the line section taken along the red line in Fig. 1(h) as well as the Fourier-transformed (FT-)QPI map, both displayed in Fig. 1(k), confirm the very low intensity of scattering events at this bias voltage. Note that this backscattering vector $\mathbf{q}_{1}$ is completely prohibited in the pristine material since reversing the wave vector would require a spin flip, a mechanism forbidden as long as time-reversal symmetry is preserved.

In contrast, very strong scattering is observed in the QPI map recorded at $E-E_{\mathrm{F}}=-10 \mathrm{meV}[$ Fig. $1(\mathrm{~g})]$, where the CEC becomes hexagonal. Intense modulations of the $d I / d U$ signal can be recognized in the line profile [see Fig. 1(j)]. The FT-QPI map reveals the simultaneous existence of two scattering vectors, both of which-as a result of the hexagonal symmetry-appear in six equivalent directions, represented here by six (very weak) inner spots in the $\overline{\Gamma M}$ directions and six (stronger) outer spots along the $\overline{\Gamma K}$ directions. The scattering channel along $\overline{\Gamma M}\left(\mathbf{q}_{2}\right)$ is routinely found on TIs $[8,16]$. Its appearance is related to the warping term, which allows one to effectively scatter between next-nearest-neighbor segments of a CEC because of their parallel spin polarization. This channel is rather weak at the energy discussed here, $E-E_{\mathrm{F}}=$ $-10 \mathrm{meV}$, since nesting along $\mathbf{q}_{2}$ is still rather poor [see scheme in Fig. 1(d)]. In contrast, high-intensity spots are visible along $\overline{\Gamma K}$, which cannot be found on pristine TIs. Their observation implies that time-reversal symmetry is broken [17] due to the presence of the Mn adatoms, which were recently suggested to be magnetically coupled by the TI's Dirac fermions [13] (see XMCD discussion below). Further raising the energy to $E-E_{\mathrm{F}}=+70 \mathrm{meV}$ leads to a weaker modulation of the $d I / d U$ signal (see scale bars of Fig. 1). This finding is confirmed by the FT-QPI map shown in Fig. 1(i), which only reveals six weak spots along the $\overline{\Gamma M}$ direction corresponding to $\mathbf{q}_{2}$.

Interestingly, closer inspection of the real-space images reveals that once backscattering channels are opened, coherent waves propagate over distances larger than $30 \mathrm{~nm}$ without any significant intensity loss [cf. line section of Fig. 1(j)]. Due to the absence of a decay, we are not able to quantify the relaxation length by fitting with an exponential function as in Ref. [18]. It is quite obvious, however, that the lower bound exceeds hundreds of nanometers. Furthermore, although point-shape scattering centers should result in spherical waves, our experimental data presented in Fig. 1(g) indicate that the charge density oscillations remain highly focused over mesoscopic distances well beyond the atomic scale. These observations provide compelling evidence that Diracfermion-mediated interactions in TIs are highly anisotropic, as predicted theoretically [19].
A priori, the exact mechanisms that lead to the emergence of highly focused and anisotropic QPI patterns are not evident. Good nesting is known to trigger the focusing effect [5,20]. In the present case, this condition is fulfilled by large portions of the hexagonal FS. This configuration supports additional scattering vectors [see Fig. 1(d)] with $\mathbf{v}_{\mathbf{k}} \approx-\mathbf{v}_{\mathbf{k}^{\prime}}\left(\mathbf{v}_{\mathbf{k}}\right.$ is the group velocity), such as $\mathbf{q}_{1}^{\prime}$ or $\mathbf{q}_{1}^{\prime \prime}$, which-in contrast to $\mathbf{q}_{1}$ - are not strictly forbidden by time-reversal symmetry but strongly suppressed by the almost opposite spin projection of initial and final states. Their absence on pristine TIs proves the important role played by magnetic perturbations which we will analyze in detail. As we will show by means of ab initio and model-Hamiltonian simulations, the simultaneous action of both FS nesting and the presence of magnetic scatterers is required to correctly explain our experimental findings.

In our simulations, we examine an extension of the widely used joint density of states approach [7,21] to approximate FT-QPI data, which is defined as

$$
\operatorname{exJDOS}(\mathbf{q} ; E)=\int A_{\mathbf{k}}^{\text {surf }}(E) M_{\mathbf{k}, \mathbf{k}+\mathbf{q}}(E) A_{\mathbf{k}+\mathbf{q}}^{\text {surf }}(E) d^{2} \mathbf{k},
$$

at energy $E$, involving the spectral density $A_{\mathbf{k}}^{\text {surf }}(E)$ integrated in the spatial region between tip and sample surface, and the matrix element $M_{\mathbf{k k}^{\prime}}(E)=P_{\mathbf{k k}^{\prime}}(E) \gamma_{\mathbf{k k}^{\prime}}(E)$. Here, $P_{\mathbf{k k}^{\prime}}(E)$ is the scattering rate (including suppression of time-reverse scattering) and $\gamma_{\mathbf{k k}^{\prime}}(E)=1-\cos \left(\mathbf{v}_{\mathbf{k}}, \mathbf{v}_{\mathbf{k}^{\prime}}\right)$ accounts for the fact that STM probes standing waves, i.e., states with opposite group velocities are favored, in the spirit of the stationary phase approximation. $A_{\mathbf{k}}^{\text {surf }}(E)$ and $\gamma_{\mathbf{k k}^{\prime}}(E)$ are derived from the band structure, while $P_{\mathbf{k k}^{\prime}}(E)$ is calculated by the golden rule from the $T$ matrix of the impurity obtained by a calculation of the impurity Green function.

Our density-functional-theory (DFT) calculations are based on the local density approximation [22]. We employ the Korringa-Kohn-Rostoker (KKR) Green-function method for the calculation of the impurity's electronic structure and scattering properties ( $T$-matrix and surface-state scattering rate). $\mathrm{The} \mathrm{Bi}_{2} \mathrm{Te}_{3}$ surface is modeled by a six-quintuple layer (QL) film. Figure 2 summarizes a number of KKR-based numerical experiments showing the exJDOS at different energies [corresponding to warped (left) or hexagonal (right) CEC] and induced by different defects/impurities [top: nonmagnetic Te vacancy; middle: single magnetic Mn impurity $\left(4.4 \mu_{\mathrm{B}}\right)$ substituting Te; bottom: magnetic Mn trimer filling three Te vacancies in a FM configuration $\left(13.1 \mu_{\mathrm{B}}\right)$ ]. If backscattering (green arrows) is present, i.e., a situation observed experimentally in Fig. $1(\mathrm{~g})$, we expect a high exJDOS $(\mathbf{q} ; E)$ intensity along the $\overline{\Gamma K}$ directions. Evidently this is only the case for Fig. 2(f), i.e., when the nesting condition is fulfilled and a very strong impurity magnetic moment $\left(13.1 \mu_{\mathrm{B}}\right.$ of the $\mathrm{Mn}$ trimer) is present. Note that the latter can only be reached with strong FM correlations. Other configurations (not shown) with the atomic moments within the Mn trimer oriented along different directions, such as $\uparrow \uparrow \downarrow$ (resulting in a magnetic moment of $4.4 \mu_{\mathrm{B}}$ ) or three in-plane-oriented spins in a $120^{\circ}$ configuration $\left(0 \mu_{\mathrm{B}}\right)$, also show a negligible backscattering intensity. The relative intensity of the Te vacancy, the single $\mathrm{Mn}$ atom $\left(\mathrm{Mn}_{1}\right)$, and $\mathrm{Mn}$ trimers with different spin configurations [in-plane $120^{\circ}$, antiferromagnetic (AFM), and FM] is depicted 

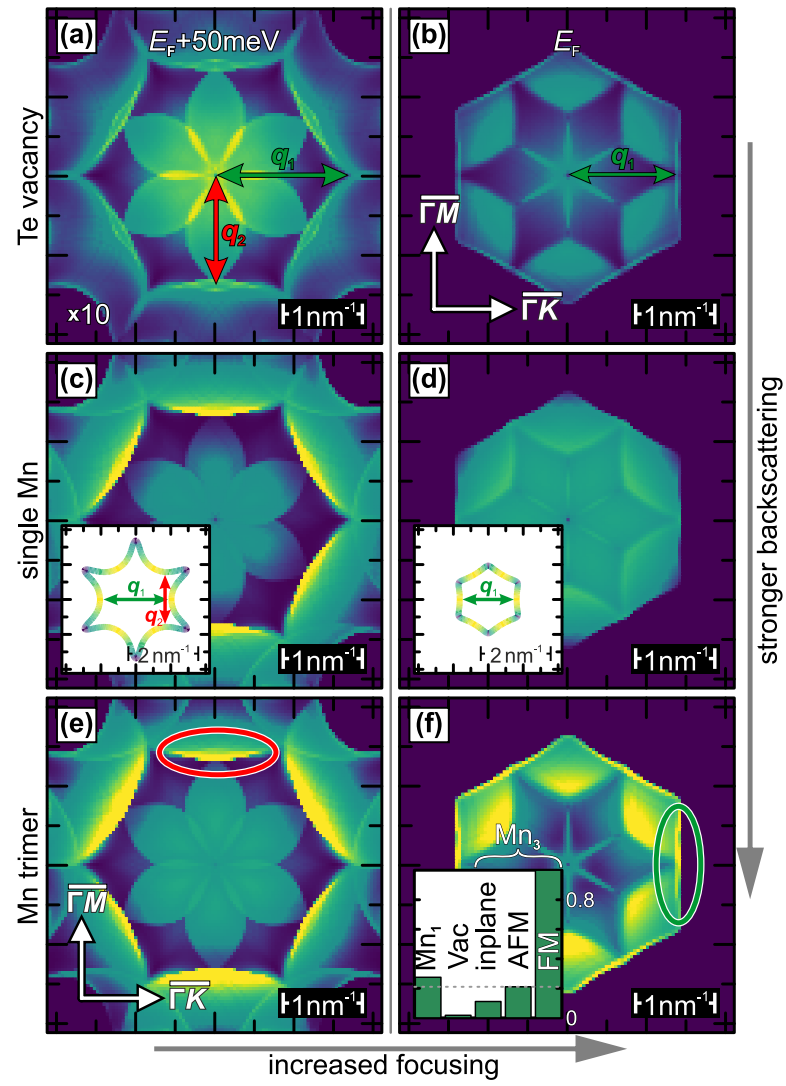

FIG. 2. Simulated exJDOS images for energies (a),(c),(e) $E=$ $E_{F}+50 \mathrm{meV}$ and (b),(d),(f) $E=E_{F}$ as generated by a Te vacancy (top row), a single $\mathrm{Mn}$ atom at a Te vacancy site (middle), and a Mn trimer (bottom). With the exception of (a) (contrast tenfold magnified), the color scale is the same in all panels. Red $(\overline{\Gamma M}$ direction) and green ( $\overline{\Gamma K}$ direction) ellipses highlight exJDOS features associated to conventional scattering and backscattering, respectively. Insets in (c) and (d) show isoenergy contours for the different energies color coded by the states' surface localization (yellow: $>80 \%$ in first QL; dark blue: $\sim 60 \%$ in first QL). In the inset in (f), the relative magnitude of the backscattering signal is compared for five different impurity and spin configurations.

in the histogram shown in the inset of Fig. 2(f). Indeed, our $a b$ initio DFT calculations performed for the Mn trimer show that the FM state has the lowest total energy as compared to the other configurations discussed above. We speculate that this may be the result of indirect RKKY-type coupling mediated by the Dirac fermions which exhibit a Fermi wavelength of approximately $7 \mathrm{~nm}$, i.e., well above the average Mn-Mn spacing of about $3 \mathrm{~nm}$ (see Supplemental Material [14]), making the interaction always FM as predicted in Ref. [17].

In parallel, we performed model calculations based on the Hamiltonian of Lee et al. [23]. As described in the Supplemental Material [14], the model was extended by exchange-interaction terms to represent (i) the scattering by Mn moments, as well as (ii) the possibility of a uniform magnetization of the surface state caused by FM coupling among the Mn defects. These model calculations, in agreement with the $a b$ initio results, provide further evidence that the experimentally observed standing wave patterns arise from the combined action of ferromagnetically coupled Mn atoms and the hexagonally shaped CEC supporting the focusing effect.

Achieving such a high magnetic moment in a Mn-doped system is possible only if Mn atoms couple FM. However, both bulk Mn [24] and Mn nanostructures [25] are known to exhibit an AFM ground state. In order to directly determine the magnetic moment and configuration of $\mathrm{Mn}$ on $\mathrm{Bi}_{2} \mathrm{Te}_{3}$, we have performed XMCD measurements at the BOREAS beam line of the Alba synchrotron facility. By measuring the photo-induced sample drain current, XAS were recorded in the total electron yield mode with left $\left(I^{-}\right)$and right $\left(I^{+}\right)$circularly polarized photons and in the presence of an external magnetic field. Figure 3(a) (top panel) reports the XAS recorded at the Mn $L_{2,3}$ edges with normal and grazing $x$-ray incidence, at a Mn coverage of about $0.016 \mathrm{ML}$. The data were taken at $T=2.5 \mathrm{~K}$ and in a magnetic field of $6 \mathrm{~T}$ applied along the photon beam direction. The spectra are characteristic of $\mathrm{Mn}$ atoms in a configuration close to $d^{5}$, as previously observed for $\mathrm{Mn}$ doped into the bulk of $\mathrm{Bi}_{2} \mathrm{Te}_{3}$ [26].

The XMCD, calculated as $I^{-}-I^{+}$and shown in the bottom panel of Fig. 3(a), highlights the considerable magnetic polarization of the Mn surface dopants. Figure 3(b) displays the magnetization cycles, recorded on the same sample at $T=2.5 \mathrm{~K}$, at normal (green line) and grazing (yellow line) incidence, by following the XMCD magnitude at the $\mathrm{Mn} L_{3}$ edge (full circles) as a function of the applied magnetic field. Both the larger slope in $M(H)$ at small fields and the saturation at large fields in normal-incidence geometry indicate an outof-plane magnetic anisotropy for $\mathrm{Mn}$ on $\mathrm{Bi}_{2} \mathrm{Te}_{3}$. The saturation magnetization (in $\mu_{\mathrm{B}}$ per $\mathrm{Mn}$ atom) can be evaluated by applying the XMCD sum rules [27,28] to the data of Fig. 3(a). Independent information on the magnitude of the fluctuating total moments is contained in the shape of the magnetization isotherms, strongly determining their slopes near $\mu_{0} H=0$. An analysis in terms of classical Langevin paramagnetism augmented with a uniaxial magnetic anisotropy term [29] yields an effective value of the (total) saturation moment $M_{\text {sat }}$ and the associated magnetic anisotropy energy.

Figure 3(c) shows the coverage dependence of $M_{\text {sat }}$ between 0.005 and $0.03 \mathrm{ML} \mathrm{Mn}$. At the lowest coverage of $0.005 \mathrm{ML}$, we obtain an effective moment near $5 \mu_{\mathrm{B}}$, coinciding with the saturation moment of $\mathrm{Mn}$ atoms with $d^{5}$ configuration. In agreement with expectation, the adatoms can be considered as magnetically independent in this limit. Already at a coverage of $0.008 \mathrm{ML}$, however, a significant enhancement of the effective magnetic moment becomes evident. Further increasing the coverage to $0.016 \mathrm{ML}$ leads to an effective moment of almost $7 \mu_{\mathrm{B}}$. Such a large value cannot be ascribed to single $\mathrm{Mn}$ atoms. Instead, it implies the existence of FM interactions among neighboring $\mathrm{Mn}$ atoms.

We envision that the exchange coupling between $\mathrm{Mn}$ adatoms may be mediated by the surface electron gas of $\mathrm{Bi}_{2} \mathrm{Te}_{3}$. We speculate that-although FM correlations are present-thermal fluctuations and disorder are too strong to establish stable FM order. Nevertheless, assemblies of $\mathrm{Mn}$ atoms with small enough Mn-Mn separation will exhibit sufficiently strong magnetic interactions to couple their individual moments to a macrospin, resulting in the enhanced susceptibility characteristic for superparamagnets [see 

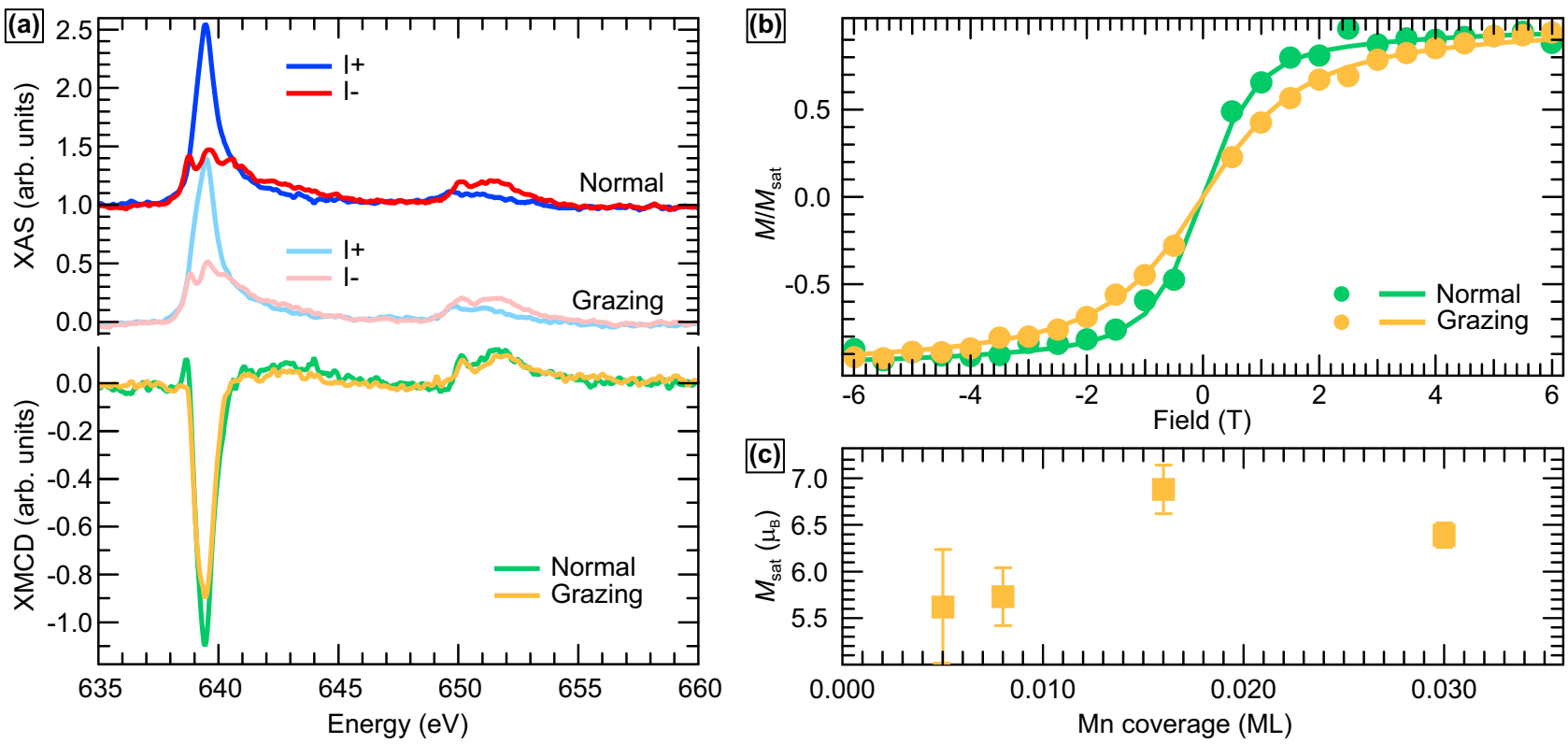

FIG. 3. (a) Mn $L_{2,3}$-edges x-ray absorption spectra (XAS; top panel) and x-ray magnetic circular dichroism (XMCD; bottom panel) signal of $0.016 \mathrm{ML} \mathrm{Mn}$ on $\mathrm{Bi}_{2} \mathrm{Te}_{3}$, obtained at normal and grazing incidence, with $B=6 \mathrm{~T}$ and $T=2.5 \mathrm{~K}$. (b) Corresponding magnetization cycles, recorded at the XMCD maximum at the $\mathrm{Mn} L_{3}$ edge. (c) Mn-coverage dependence of the saturation magnetization extracted from the recorded magnetization cycles.

Fig. 3(b)]. This interaction may, for example, be mediated by the topological surface state via RKKY interaction. In fact, an analysis of the Mn-Mn interatomic spacing in STM images shows a distribution which peaks between 1 and $3 \mathrm{~nm}$ (see Supplemental Material [14]), i.e., at a distance well below the Fermi wavelength of $\mathrm{Bi}_{2} \mathrm{Te}_{3}$, with some $\mathrm{Mn}$ atoms found in very close proximity $(\leqslant 5 \AA)$, consistent with Te-Te nearestneighbor distances. Since XMCD spatially averages over a macroscopic sample area, a value of $7 \mu_{\mathrm{B}}$ implies the existence of assemblies with considerably larger magnetic moments. This result is consistent with the theoretical finding that units composed of at least three ferromagnetically interacting $\mathrm{Mn}$ atoms are required to activate the scattering channel along the $\overline{\Gamma K}$ direction.

Our findings provide evidence that through electron focusing, quantum coherent information can be transferred in topological insulators over distances of more than $30 \mathrm{~nm}$, making it compatible with device dimensions and thereby paving the way to design experiments and devices based on spin quantum coherent phenomena in this fascinating class of materials. More generally, they provide evidence that through appropriate band engineering in materials with spin-split states, many interesting phenomena may appear, with important implications for spintronics.

This work was performed within the DFG-funded SPP 1666 (Projects No. BO 1468/21-1 and No. MA 4637/3-1). P.R., N.H.L., P.M., and S.B. acknowledge financial support from the VITI project of the Helmholtz Association as well as computational support from the JARA-HPC Supercomputing Centre at RWTH Aachen. M.B. acknowledges supported through SFB 1170 "ToCoTronics" (Project No. A02). The work was partially supported by the Italian Government (MIUR Progetto Premiale "Materiali e disposivi magnetici e superconduttivi per sensoristica e ICT"). M.A.V., S.G., and A.M. acknowledge support from the Ministerio de Ciencia e Innovacion (Grant No. MAT2013-46593-C6-5-P), the Severo Ochoa Program (MINECO, Grant No. SEV-2013-0295), and Agéncia de Gestiò d'Ajuts Universitaris i de Recerca (Grant No. 2014 SGR715)
[1] M. F. Crommie, C. P. Lutz, and D. M. Eigler, Nature (London) 363, 524 (1993).

[2] H. C. Manoharan, C. P. Lutz, and D. M. Eigler, Nature (London) 403, 512 (2000).

[3] C. R. Moon, L. S. Mattos, B. K. Foster, G. Zeltzer, W. Ko, and H. C. Manoharan, Science 319, 782 (2008).

[4] L. Zhou, J. Wiebe, S. Lounis, E. Vedmedenko, F. Meier, S. Blügel, P. H. Dederichs, and R. Wiesendanger, Nat. Phys. 6, 187 (2010).

[5] A. Weismann, M. Wenderoth, S. Lounis, P. Zahn, N. Quaas, R. G. Ulbrich, P. H. Dederichs, and S. Blügel, Science 323, 1190 (2009).
[6] M. Z. Hasan and C. L. Kane, Rev. Mod. Phys. 82, 3045 (2010).

[7] P. Roushan, J. Seo, C. V. Parker, Y. S. Hor, D. Hsieh, D. Qian, A. Richardella, M. Z. Hasan, R. J. Cava, and A. Yazdani, Nature (London) 460, 1106 (2009).

[8] T. Zhang, P. Cheng, X. Chen, J.-F. Jia, X. Ma, K. He, L. Wang, H. Zhang, X. Dai, Z. Fang et al., Phys. Rev. Lett. 103, 266803 (2009).

[9] M. König, S. Wiedmann, C. Brüne, A. Roth, H. Buhmann, L. W. Molenkamp, X.-L. Qi, and S.-C. Zhang, Science 318, 766 (2007).

[10] Y. L. Chen, J. G. Analytis, J.-H. Chu, Z. K. Liu, S.-K. Mo, X. L. Qi, H. J. Zhang, D. H. Lu, X. Dai, Z. Fang, S. C. Zhang, 
I. R. Fisher, Z. Hussain, and Z.-X. Shen, Science 325, 178 (2009).

[11] L. Fu, Phys. Rev. Lett. 103, 266801 (2009).

[12] Y. Okada, C. Dhital, W. Zhou, E. D. Huemiller, H. Lin, S. Basak, A. Bansil, Y.-B. Huang, H. Ding, Z. Wang, S. D. Wilson, and V. Madhavan, Phys. Rev. Lett. 106, 206805 (2011).

[13] P. Sessi, F. Reis, T. Bathon, K. A. Kokh, O. E. Tereshchenko, and M. Bode, Nat. Commun. 5, 5349 (2014).

[14] See Supplemental Material at http://link.aps.org/supplemental/ 10.1103/PhysRevB.94.075137 for details of the experimental and theoretical procedures and results.

[15] Q. Liu, X.-L. Qi, and S.-C. Zhang, Phys. Rev. B 85, 125314 (2012).

[16] P. Sessi, M. M. Otrokov, T. Bathon, M. G. Vergniory, S. S. Tsirkin, K. A. Kokh, O. E. Tereshchenko, E. V. Chulkov, and M. Bode, Phys. Rev. B 88, 161407 (2013).

[17] Q. Liu, C.-X. Liu, C. Xu, X.-L. Qi, and S.-C. Zhang, Phys. Rev. Lett. 102, 156603 (2009).

[18] L. Bürgi, O. Jeandupeux, H. Brune, and K. Kern, Phys. Rev. Lett. 82, 4516 (1999).

[19] R. R. Biswas and A. V. Balatsky, Phys. Rev. B 81, 233405 (2010).

[20] S. Lounis, P. Zahn, A. Weismann, M. Wenderoth, R. G. Ulbrich, I. Mertig, P. H. Dederichs, and S. Blügel, Phys. Rev. B 83, 035427 (2011).
[21] A. Eich, M. Michiardi, G. Bihlmayer, X.-G. Zhu, J.-L. Mi, B. B. Iversen, R. Wiesendanger, P. Hofmann, A. A. Khajetoorians, and J. Wiebe, Phys. Rev. B 90, 155414 (2014).

[22] S. H. Vosko, L. Wilk, and M. Nusair, Can. J. Phys. 58, 1200 (1980).

[23] W.-C. Lee, C. Wu, D. P. Arovas, and S.-C. Zhang, Phys. Rev. B 80, 245439 (2009).

[24] A. C. Lawson, A. C. Larson, M. C. Aronson, S. Johnson, Z. Fisk, P. C. Canfield, J. D. Thompson, and R. B. Von Dreele, J. Appl. Phys. 76, 7049 (1994).

[25] P. Sessi, N. P. Guisinger, J. R. Guest, and M. Bode, Phys. Rev. Lett. 103, 167201 (2009).

[26] M. D. Watson, L. J. Collins-McIntyre, L. R. Shelford, A. I. Coldea, D. Prabhakaran, S. C. Speller, T. Mousavi, C. R. M. Grovenor, Z. Salman, S. R. Giblin, G. van der Laan, and T. Hesjedal, New J. Phys. 15, 103016 (2013).

[27] B. T. Thole, P. Carra, F. Sette, and G. van der Laan, Phys. Rev. Lett. 68, 1943 (1992).

[28] P. Carra, B. T. Thole, M. Altarelli, and X. Wang, Phys. Rev. Lett. 70, 694 (1993).

[29] P. Gambardella, S. Rusponi, M. Veronese, S. S. Dhesi, C. Grazioli, A. Dallmeyer, I. Cabria, R. Zeller, P. H. Dederichs, K. Kern et al., Science 300, 1130 (2003). 